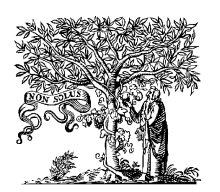

ELSEVIER

\title{
A laboratory assessment of wetness sensors for leaf, fruit and trunk surfaces
}

\author{
Ricardo A.C. Miranda ${ }^{\mathrm{a}, *}$, Trevor D. Davies ${ }^{\mathrm{b}, 1}$, Sarah E. Cornell ${ }^{\mathrm{b}}$ \\ a Centro de Pesquisas do Cacau, Recursos Ambientais-Climatologia, Caixa Postal 7, Itabuna, Bahia, 45600-000, Brazil \\ ${ }^{\mathrm{b}}$ University of East Anglia, School of Environmental Sciences, Norwich, NR4 7TJ, UK
}

Received 21 June 1999; received in revised form 24 January 2000; accepted 24 January 2000

\begin{abstract}
A laboratory assessment has been made of the performance and applicability of several different conductimetric wetness detectors for use in monitoring water distribution on different plant surfaces in a tree canopy. Leaf surfaces were monitored using parallel electrode clip sensors and grid sensors. Fruit and trunk wetness was monitored using parallel electrode sensors encircling fruit and trunk samples. Modifications to the fruit sensors to facilitate fitting and reduce the risk of epidermal damage have been investigated. Generally, the simplest parallel electrode designs performed better than the sensors with intended design refinements, in terms of instantaneous signal onset sensitivity, the matching of sensor signal profiles with weighed water loss profiles, and the accuracy of the determination of wetness persistence on the sampled surfaces. Factors affecting the drying profiles obtained using the sensors include the previous wetting history, the distribution of water on the surfaces, and the surface texture. (C) 2000 Elsevier Science B.V. All rights reserved.
\end{abstract}

Keywords: Leaf wetness duration; Wetness sensors; Conductivity; Evaporation; Water interception

\section{Introduction}

Microclimatic conditions within a plant canopy strongly influence its biological productivity. For many plants, this impacts on the economic yield. Commercial growers are particularly interested in wetness, one of the fundamental variables in the within-canopy microclimate, because the persistence

\footnotetext{
* Corresponding author. Present address: Universidade do Estado do Rio de Janeiro, Centro de Tecnologia e Ciências, Instituto de Geociências, departamento de Climatologia e Meteorologia, Rua São Francisco Xavier, No. 524, 5/4006, B1 F, Maracanã, Rio de Janeiro, Brasil, 20550-013. Fax: +21-587-7350.

E-mail addresses: rac_miranda@hotmail.com (R.A.C. Miranda), t.davies@uea.ac.uk (T.D. Davies), s.cornell@uea.ac.uk (S.E. Cornell)

${ }^{1}$ Fax: +44-1603-507719.
}

of wetness on plant surfaces is associated with the incidence and spread of sporulate fungal pathogens, for which water is a major vector (e.g. Chakraborty et al., 1990; Zehr et al., 1996; Hartman et al., 1999; Thakur and Khosla, 1999). Further economic impacts result from the presence of water in the canopy even when pathogens are not involved (for example, mechanical damage, and damage to leaves and fruit by the wet deposition of acid).

The quantification of wetness distribution in plant canopies provides input data for modelling plant damage and diseases (Huber and Wehrlen, 1988; Duthie, 1997; Wilson et al., 1999). For these models, measures of the distribution of incident rainfall need to be supplemented by measurements of wetness persistence on plant faces. Sensors can detect wetness electronically either on artificial surfaces placed within or outside 
a plant canopy (e.g. Fisher et al., 1992; Penrose and Nicol, 1996; deWolf and Francl, 1997) or in situ on plant surfaces (Häckel, 1974; Weiss et al., 1988; Wei et al., 1995). Most in situ studies have focused on leaf surfaces, as they provide most of the canopy's surface area on which water may be deposited. These studies have shown that wetness is highly variable within the canopy (Huber and Wehrlen, 1988). Since it is evident that micro-scale variability in canopy conditions can have a large impact on plant health and productivity (Chakraborty et al., 1990; Penrose and Nicol, 1996), extensive sampling is needed to represent the wetness distribution adequately (Weiss and Hagen, 1983; Penrose and Nicol, 1996).

Few researchers have measured or modelled wetness persistence on non-foliar surfaces (James et al., 1984; Rudgard and Butler, 1987), although this approach could address the problems of heterogeneity in the canopy. An understanding of fruit surface wetness is critical in modelling fruit damage and disease, yet it is unclear how well this may be obtained by extrapolation from data obtained from leaf surfaces, or from out-of-canopy plate sensor surfaces. This is because fruits, with their large mass and high water content, have a higher specific heat capacity than leaves (Sutton et al., 1988): they undergo slow heat exchange with the surrounding atmosphere because of their low surface area to mass ratio; and their greater density means they are less subject to the mechanical action of winds, which effectively shakes the water off leaves (Ward, 1988). Fruits and leaves differ in their surface characteristics, which affect water distribution (e.g. waxiness and fuzziness are natural adaptations protecting fruits from water buildup).

The tree trunk is seen predominantly as a conduit for water flow. However, a few commercially important fruit trees have fruit growth from the trunk, with cocoa being a prime example, and an understanding of their vulnerability to water-borne pathogens requires an understanding of wetness distribution on the trunk as well as on the other surfaces. Stemflow is known to be highly variable between rain events (Miranda and Butler, 1986; Aboal et al., 1999). Unlike fruit and leaves, which are impermeable to water, the porosity of bark means that ambient humidity conditions and previous wetness history have a significant influence on the drying times of surface wetness. This means, of course, that the persistence of trunk wetness is likely to exhibit a multimodal distribution.

There are difficulties in characterising wetness patterns in a system which is naturally highly variable. Many sensor types are used, including commercially obtained radiometric sensors, artificial surface sensors, and electronic (resistance) sensors, which give variable and non-linear responses depending on factors such as the distribution rather than the quantity of water present (Weiss and Hagen, 1983; Sutton et al., 1984; Huber and Wehrlen, 1988). Several studies have compared sensors (Barthakur, 1985, 1987; Armstrong et al., 1993), which are continuously modified and improved (Fisher et al., 1992), but one can broadly conclude that for any given application, an empirical process of sensor selection, construction and optimisation still needs to be carried out.

Here we report the results of an experimental investigation of electrical resistance wetness sensors designed to detect the presence of water on leaf, fruit and trunk surfaces, in order to provide wetness distribution and persistence data on a small scale for use in predictive modelling of an individual tree canopy.

\section{Methods and procedure}

The primary aim of this study was to evaluate the use and applicability of sensors of simple and economical design on the major types of plant surface within a tree canopy. In practice, the ease of use and the unit cost of sensors rank as highly in the list of priorities as the sensitivity and accuracy of the sensors. The sensors tested here can easily and routinely be used in agricultural plantations where more complex or costly methods of wetness monitoring may be inappropriate. Ideally, a wetness sensor should not interfere with the natural cycling of water in the canopy, and its output should unambiguously reflect the local conditions. Of course, in practice, modifications that reduce the sensor's impact on the natural cycling of water (such as sensor size reduction, or removal from the surfaces themselves) are likely to reduce the accuracy of the sensor's representation of its surrounding conditions. This is particularly true of sensors on leaves, which have complex surface texture, a large surface area, and relatively small mass. In this study, two leaf wetness sensors of different designs are com- 


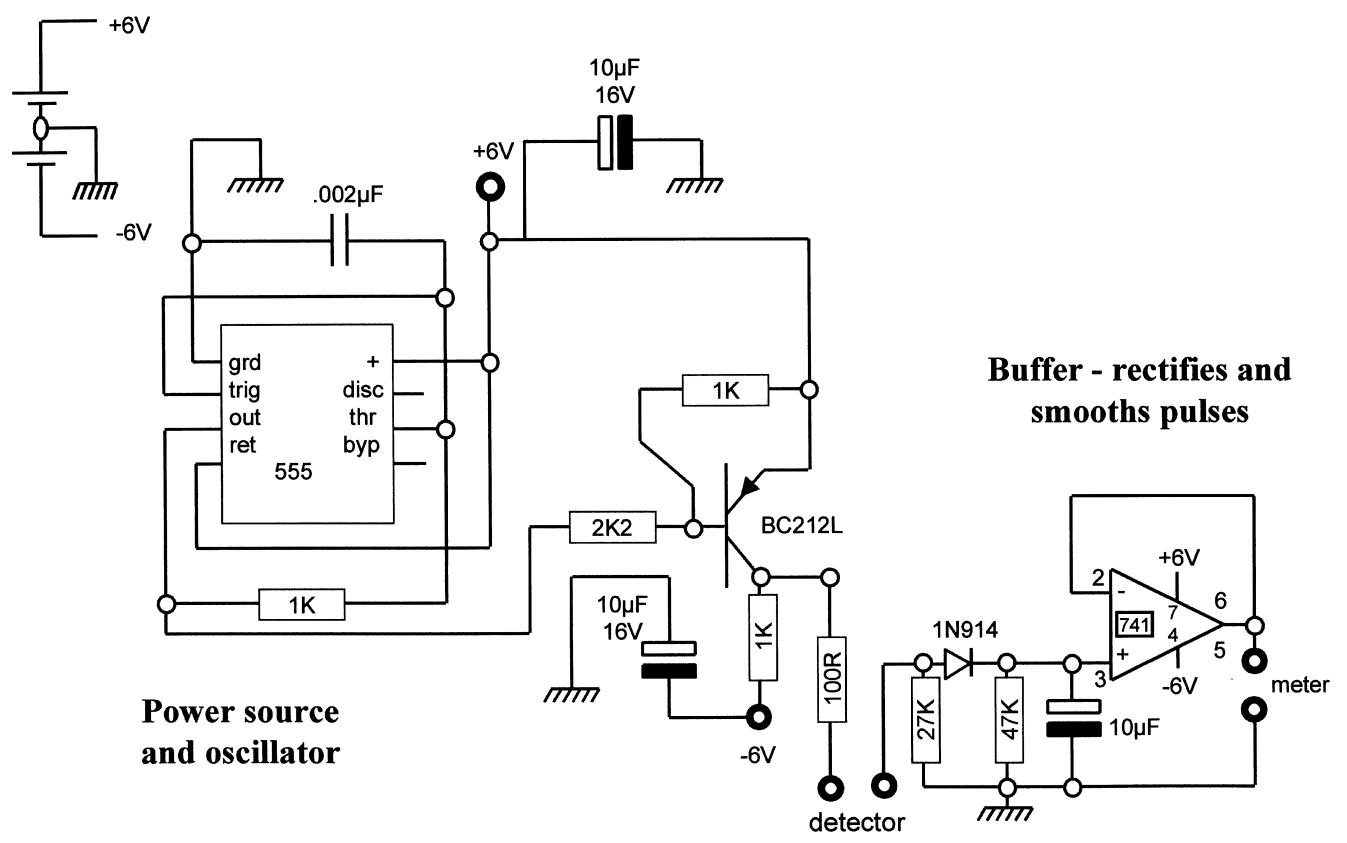

Fig. 1. Circuit schematic for the electronic wetness sensors.

pared, with particular emphasis on interference and accuracy. In addition, two types of wetness sensor for fruit and a trunk sensor are assessed.

The evaluation of the electrical wetness sensors was carried out empirically in the laboratory, where the sensor output was compared with measured water mass loss and with visual observations of wetness on the plant surfaces. The objective was to investigate the response of the sensors to the range of exposures to wetness typical in the field.

\subsection{Sensor descriptions}

All the sensors (Fig. 1) responded to the change in conductance associated with the presence of water on a surface. A $12 \mathrm{~V}$ DC power supply energised the sensors, via a $20 \mathrm{~Hz}$ oscillator, feeding alternating pulses to one of the electrodes of the sensor. The baseline voltage for all the dry sensors when fitted to the plant surfaces was $0 \pm 5 \mathrm{mV}$. When water was present on the sample surface, conductance increased with the amount of water between the electrodes, and the resulting voltage change was detected, rectified and smoothed, and recorded at $5 \mathrm{~min}$ intervals using a 16-channel data logger (Campbell Scientific, Campbell 21X, Leicester, UK). Water mass loss measurements and visual assessments of wetness were made at the same times. The response varied from sensor to sensor, as different types of sensors had different electrode contact areas and the signals were not attenuated nor amplified. The sensor response outputs varied from -2 to $0 \mathrm{mV}$ for a dry sensor to $1-3 \mathrm{~V}$ for a completely wet sensor (i.e. where a continuous film or puddle of water completely bridged the electrodes). The logged data were retrieved using a 486 SX-33 MHz Toshiba notebook and software provided by Campbell Scientific.

Two types of leaf sensors (Fig. 2a) were purposemade at the University of East Anglia's School of Environmental Sciences. The first was a grid wetness sensor adapted from specifications described by Weiss and Lukens (1981) and Weiss and Hagen (1983). The second was a clip sensor based on designs of Sutton et al. $(1984,1988)$.

The grid wetness sensor consisted of a parallel array of nickel chromium wires $(0.2 \mathrm{~mm}$ diameter) woven tightly through two perforated plastic boards at a spacing of $5 \mathrm{~mm}$ between wires, so that each wire was 
a. Leaf wetness sensors

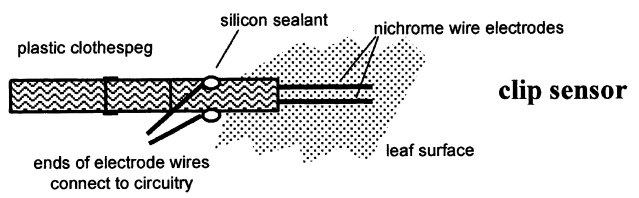

connect to circuitry

b. Fruit wetness sensors
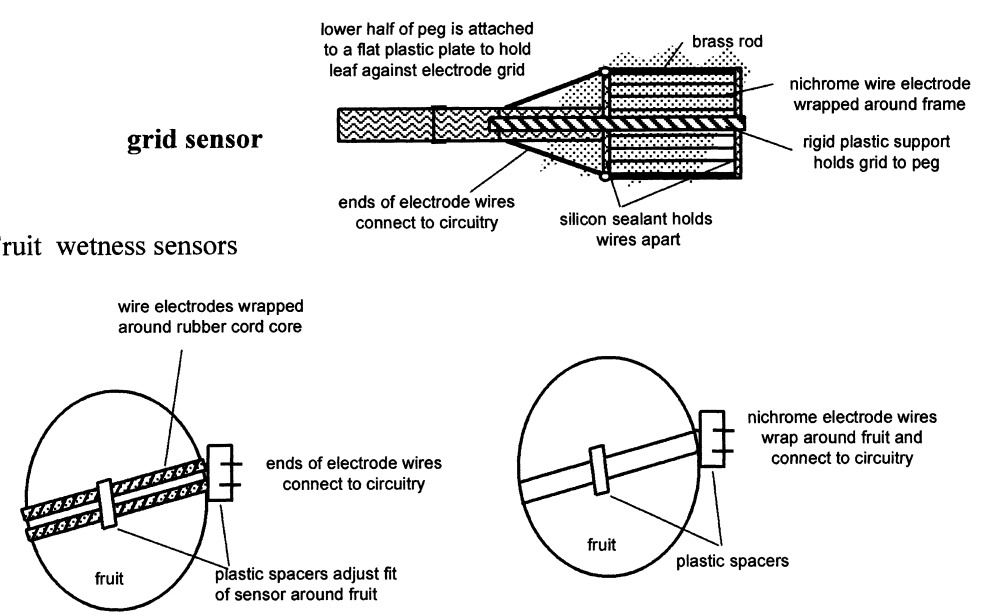

spiral-bound rubber

bare wire electrode sensor electrode sensor

c. Trunk wetness sensor

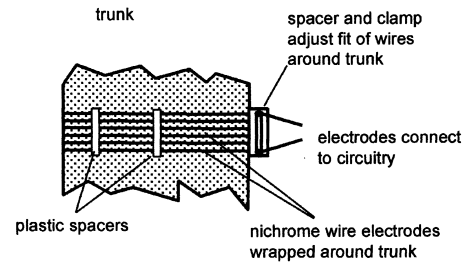

Fig. 2. Application of sensors to different types of plant surfaces: (a) shows plan views of the parallel electrode clip sensor and the grid sensor used for leaf surface wetness detection; the clip sensor parallel electrodes are the same on the underside of the sampled leaf. The grid sensor has a non-conducting plate on the underside, which sandwiches the leaf against the electrode grid frame. (b) Shows the spiral bound rubber sensor and the parallel bare electrode sensor for fruit; and (c) shows the parallel electrode sensor for trunk wetness.

electrically insulated from its neighbouring wires. The plastic boards were supported by a rigid rectangular frame, formed from two brass rods. The total frame size was $3.0 \mathrm{~cm} \times 3.0 \mathrm{~cm}$, and the frame was fixed to the front end of a plastic clip (clothespeg style clips were used). The sampled leaves were sandwiched between the grid and a slightly convex plastic board mounted on the lower half of the plastic clip.

Clip sensors (Fig. 2a) monitored the electrical conductivity between a parallel pair of electrodes resting directly on the surfaces of the apple leaves. The sensors were made of two nickel chromium wires $(0.25 \mathrm{~mm}$ diameter) mounted side by side, $2 \mathrm{~mm}$ apart, on each half of a plastic clip, extending past the front edge of the clip by about $2 \mathrm{~cm}$. The sensors were clipped onto the sampled leaves, which were held between the clip sensors' electrodes.

The fruit and trunk sensors (Fig. 2b and c) were conceptually very similar to each other. In all cases, nichrome wire electrodes encircled the sample, with the electrodes held directly on the sample surfaces. Brass fittings were used to adjust the sensors to fit 
different sized samples, and to connect the electrodes to the excitation circuit and the data logger, which recorded the flow of current between electrode contacts when water bridged them. Rudgard and Butler (1987) had previously used parallel nichrome wire electrodes for wetness detection on cocoa fruits. In this study, the sensors were tested on apple trunk sections and on apples. The trunk sensors consisted of three pairs of parallel $0.25 \mathrm{~mm}$ diameter nichrome wires, wrapped tightly around the trunk sample at about $15 \mathrm{~cm}$ from the top, separated by $10 \mathrm{~mm}$ using plastic spacers, and fastened through plastic clamps before connecting to the circuitry. The sample apple fruits were fitted with two parallel $0.25 \mathrm{~mm}$ diameter nichrome electrodes held $10 \mathrm{~mm}$ apart with spacers, encircling the samples horizontally around the widest girth of the fruits. The spiral bound rubber sensor for fruits was an adaptation designed to facilitate fitting and to minimise damage to the fruit epidermis. In this sensor, the electrodes consisted of $0.25 \mathrm{~mm}$ nickel chromium wire wrapped in a spiral around a central elastic rubber core (approximately $2.5 \mathrm{~mm}$ diameter). Like the parallel bare electrode sensors, the two electrodes were held apart at a fixed distance of $10 \mathrm{~mm}$ by plastic spacers placed at regular intervals along the length of the rubber cord.

\subsection{Procedure}

The leaf sensors were clipped directly to leaves, which were still attached to a sample section of apple tree branch. The leaves were kept turgid by the immersion of the cut end of the branch in water in a sealed plastic cylinder flask. The sensors were placed near each other at the top of the sample branch. For both sensor types, the sensors were kept steady and properly attached to the leaves by a clamp stand. Fruit sensors were fitted onto detached apples held on a clamp stand, and the trunk sensors were fitted to $25 \mathrm{~cm} \mathrm{sec-}$ tions of apple tree trunk (approximately $15 \mathrm{~cm}$ diameter). Throughout the assessment study, the electrode wires were lightly cleaned between observations using fine grade sandpaper to minimise variability between experiments due to possible dirt accumulation which could increase their resistance.

The samples were weighed on a top-weighing electronic balance (Mettler P1210, maximum weight $=1200 \mathrm{~g}$, gradations of $10 \mathrm{mg}$ ). Environmental conditions in the laboratory were much more consistent than they would be in the field: the calibration assessments were all carried out during the day, when ambient (indoor) temperatures were between 21 and $25^{\circ} \mathrm{C}$, and the samples were not exposed to large fluctuations of humidity or to much air movement. This relative consistency of environment during the sensor assessment permitted conclusions to be drawn about the intrinsic variability and applicability of the sensors which should be pertinent whatever the field conditions.

For all surfaces, ordinary tap water from the main supply was applied artificially by spraying from a misting spray bottle. For fruit and leaf surfaces, the spray was applied from a distance of $20 \mathrm{~cm}$ from the side of the samples, but for the trunk assessment, the spray was directly at the top of the trunk section, not directly at the electrodes, so that water could flow down the trunk as it does in the field. Norwich mains water typically has a conductivity of $0.6 \mu \mathrm{S} \mathrm{cm}^{-1}$, and a total ionic strength of $15 \mu \mathrm{mol}^{-1}$. This is less than the typical conductivity and total ionic strength of rainwater from the region of the experiment, which average $30 \mu \mathrm{S} \mathrm{cm}^{-1}$ and $280 \mu \mathrm{moll}^{-1}$, respectively (Cornell, 1996). The wetness (rains, dew or fog condensation) on plant surfaces in the field is variable between events, in terms of ionic strength, and also of the content of organic matter, which may be expected to alter surface properties and drying behaviour. This variability is reflected in differences in the magnitude of the sensors' response to wetting events, but does not alter the sensor's ability to detect 'dryness', defined as a return to the baseline voltage described above.

Once the sample was wetted, the change in weight was noted from the balance, and recorded at $5 \mathrm{~min}$ intervals until it returned to the initial (dry) weight. The degree of surface wetness was simultaneously assessed by visual observations at 5 min intervals, according to a single criterion classification (one way). Surface wetness was scored from 0 to 4 according to the following scale: 0 , dry surface; $1,25 \%$ of surface wet; $2,50 \%$ wet; $3,75 \%$ wet; and 4, surface totally covered with water. In practice, the limitation of this method of visual assessment is that the same wetness score on different occasions could refer to different volumes of water, because of the formation of droplets on the sample surface. 
Table 1

Summary of sensor performance

\begin{tabular}{lcll}
\hline Sensor type & $\begin{array}{l}\text { Peak response } \\
\mathrm{mV}( \pm \mathrm{SD})\end{array}$ & $\begin{array}{l}\text { Prompt }(<1 \mathrm{~min}) \text { signal } \\
\text { increase on wetting? }\end{array}$ & $\begin{array}{l}\text { Correlation }^{\mathrm{b}} \text { between voltage output and } \\
\text { water mass in drying profiles }\end{array}$ \\
\hline Leaf clip sensor & $900 \pm 180(n=26)$ & Yes & 0.65 \\
Leaf grid sensor & $2200 \pm 305(n=7)$ & $\mathrm{No}^{\mathrm{c}}$ & 0.74 \\
Fruit parallel bare electrodes sensor & $1250 \pm 225(n=16)$ & Yes & 0.55 \\
Fruit spiral-bound rubber sensor & $160 \pm 95(n=8)$ & Yes & 0.90 \\
Trunk parallel bare electrodes sensor & $1800 \pm 145(n=6)$ & Yes & 0.97
\end{tabular}

${ }^{a}$ The peak response is the average magnitude of the initial sensor voltage output when water is sprayed over the sensors fitted to the sample. The standard deviation for $n$ comparable wetting experiments carried out with each type of sensor is also given.

${ }^{\mathrm{b}}$ These are the correlation coefficients, $r$, for the water mass loss profile and the sensor voltage output for the profiles shown in Figs. 2-5.

${ }^{\mathrm{c}}$ Up to 10 min delay was observed between wetting the sample and the peak sensor response in about one third of wetting trials.

\section{Wetness assessment}

The different sensor types have been assessed with regard to various aspects of their response, as summarised in Tables 1 and 2. In the discussion that follows, the first factor taken into account was the initial response to the application of water, here termed the onset sensitivity. The sensitivity of the sensor output to differences in water volume and distribution is also discussed, since in these factors both have a significant impact on the drying process. Finally, the overview of sensor behaviour is extended to a discussion of the design implications.

\subsection{Leaf surface wetness}

The clip and grid sensors both gave a clearly detectable increase in conductance when water was applied to the sample leaves. For the clip sensors, this voltage increase was instantaneous, but for the grid sensors, a full response was often delayed by up to $10 \mathrm{~min}$. The peak onset delays were particularly evident when water was applied as a very light spray. These observations suggest that the grid sensor structures may interfere with the deposition of water on the leaf surfaces. While the peak voltage output on wetting was always distinct from the baseline dry voltage, the peaks for the fine misting trials (typical examples for both sensor types are shown in Fig. 3a) were not significantly different from heavy wetting trials (typical examples given in Fig. 3b), when larger volumes of water were applied to the leaf surface to form droplets and puddles. The peak voltage output for the clip sensors ranged from $700 \mathrm{mV}$ to $1.2 \mathrm{~V}$, while the response tended to be more closely constrained for the grid sensors, lying between 2 and $2.5 \mathrm{~V}$. For both sensor types the peak magnitude showed no evidence of a simple linear relationship to the amount of water applied, subject to an estimated minimum water coverage of $5 \mathrm{mg} \mathrm{cm}^{-2}$. The maximum voltage out-

Table 2

Comparison of drying times obtained by the different sensor ${ }^{\mathrm{a}}$ types with drying $^{\mathrm{b}}$ times determined directly by water mass loss and visual inspection

\begin{tabular}{lccc}
\hline \multirow{2}{*}{ Sensor type } & \multicolumn{2}{l}{ Drying time, min $( \pm 1 \mathrm{SD})$} & \\
\cline { 2 - 4 } & Water mass loss & Sensor voltage $^{\mathrm{c}}$ & Visual inspection \\
\hline Leaf clip sensor & $48 \pm 13$ & $48 \pm 17$ & $36 \pm 17$ \\
Leaf grid sensor & $49 \pm 10$ & $60 \pm 25$ & $49 \pm 11$ \\
Fruit parallel bare electrodes sensor & $49 \pm 18$ & $55 \pm 13$ & $45 \pm 8$ \\
Fruit spiral-bound rubber sensor & $82 \pm 12$ & $106 \pm 21$ & $83 \pm 33$ \\
Trunk parallel bare electrodes sensor & $107 \pm 17$ & $113 \pm 20$ & $93 \pm 15$ \\
\hline
\end{tabular}

a Each sensor was subject to six separate but procedurally identical wetting trials, except the leaf clip sensors, for which $n=13$.

${ }^{\mathrm{b}}$ Dryness is defined as the point at which the sample mass returns to its prewetting mass.

${ }^{\mathrm{c}}$ The sensor voltage output returns to its background level. 
a.
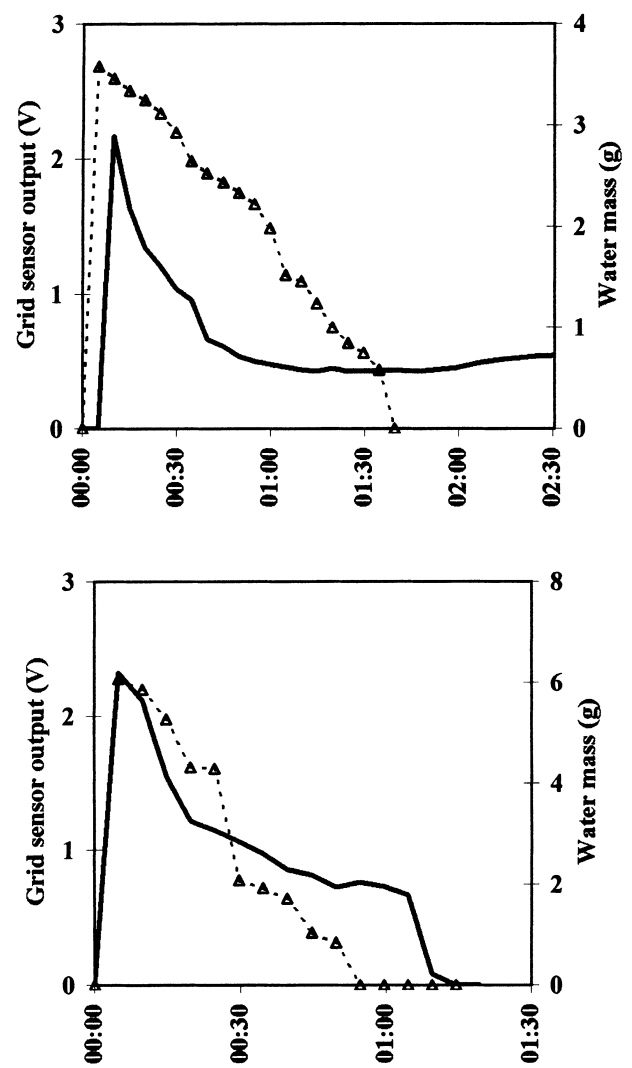

c.

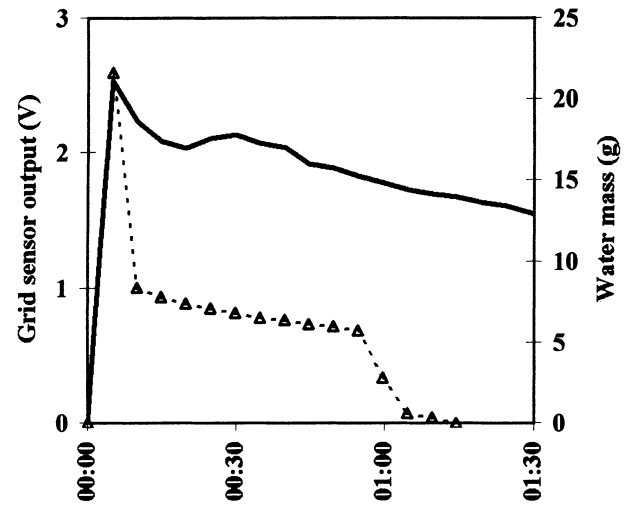

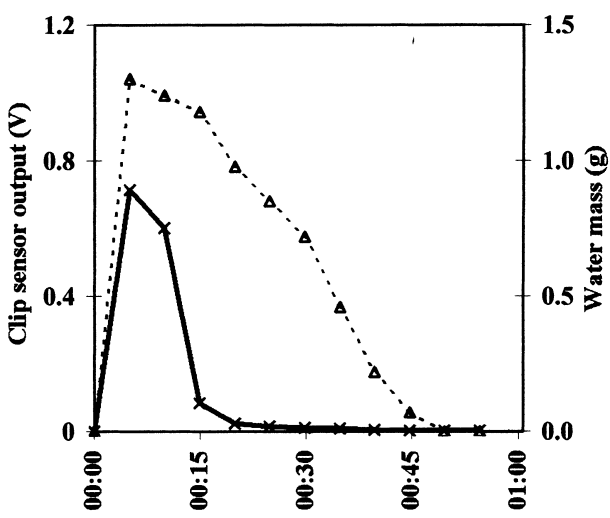
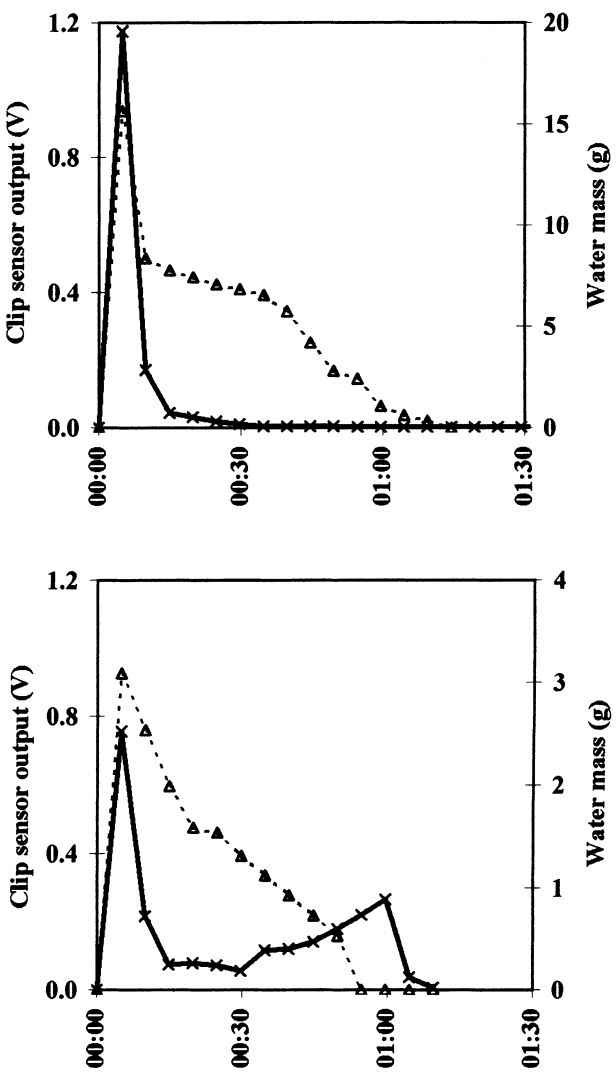

Drying time (hh:mm)

Fig. 3. Typical examples of drying profiles for artificially wetted apple leaves obtained using clip and bare-grid electrode sensors and direct water mass measurements. (a) Shows the results for light spray applied to form a thin film on the surface; (b) shows heavier wetting trials when the water coalesced into droplets; (c) shows features associated with droplets accumulating between the electrodes, with flattened profiles for the grid sensors, and irregular peaks in the profile tails for the clip sensors. 
put requires an uninterrupted film of water to fill the space between two electrodes. When the film breaks down into droplets between the electrodes, the voltage drops markedly. Clip sensors, having only two electrodes, reflect this in a sharp-peaked signal with a long tail, while the grids, with their network of electrodes and greater surface coverage, are more likely to have short-circuiting droplets that keep the voltage high, and tend to have more gradually decreasing voltage signal profiles.

The thickness of the water film has little effect on the magnitude of the peak voltage, although the water volume and distribution will, of course, have an impact on the drying time (Leclerc et al., 1985). It takes longer for the sample to return to dryness (i.e. to return to the original pre-wetting sensor voltage and sample mass) if the water forms a film than if the water is present as multiple drops, when the time for complete evaporation depends on droplet size. When the deposited water formed droplets, the drying profiles of the clip sensors sometimes showed smaller peaks in the tails after the initial decline, as water droplets could flow between the electrodes if the sensor was fitted at an angle, raising the conductance again (Fig. 3c). For grid sensors, it was often observed that while the thickness of the water film decreased (i.e. when water was being lost from the surface by evaporation, giving a decrease in measured mass), sensor readings remained constant at or near the initial onset response (Fig. 3c). These flat-topped signal profiles indicated that the sensor structures themselves may have been inhibiting evaporation. Samples were visually observed to be dry shortly before the weight measurements indicated complete evaporation, with agreement generally within 5-10 min for visual and weight-loss assessments (Table 2).

Repeated, sequential wetting of the samples and sensors, simulating intermittent field rainfall, showed that both types of electrodes detected repeat wetting events. Problems of water accumulation in the grid sensor structures were evident, with flat topped peaks and a failure to return to a complete dryness signal between wetting events, even though visual and weight measures showed dryness. These features in the sensor signal profiles were not a consequence of water absorption or damage to the leaf cuticle, as described by Ward (1988), since wiping the sensors dry with absorbent paper returned the output to the original (dry) sensor readings within 30-40 s.

On average, clip sensors agreed well with directly determined drying times, with a tendency to underestimate by about $10 \mathrm{~min}$. Although the grid sensors gave a higher voltage output than the simpler clip sensors because of the former's greater exposed electrode contact surfaces, they did not consistently show improved sensitivity as a result. The grid sensor voltage profiles yielded better correlations with the weight-loss drying profiles than the clip sensor output (Table 1 and Fig. 3), due to the greater leaf surface coverage and electrode area. On the other hand, the grid sensor output often did not return to the starting response until long after the visual and weight loss observations showed total evaporation, giving typical grid-sensor wetness durations of up to an hour longer (in some cases, a factor of two or three greater) than the direct observational methods (Table 2 and Fig. 3). A practical difficulty observed in using the grid sensors was that their electrode array creates several points where the parallel wires can trap water next to the plastic frame or between the wires, if the rigid grid is not in uniform contact with the leaf surface. This evaporates more slowly than water on the sample surface, leading to substantial over-estimation of surface wetness persistence. Other difficulties with the grid sensors were their bulk and weight. Modifications to reduce the problem of water accumulation, for instance, using electrode wires which extend only partway along the frame, would likely do little to resolve these other practical difficulties. These observations have highlighted the difficulty of developing a more sensitive and spatially representative leaf sensor, where structural complexity outweighs the desired benefits.

\subsection{Fruit surface wetness}

Both the spiral-bound rubber sensor and the bare electrodes sensor showed appropriate sensitivity to the application of water, with the larger contact area of the bare electrodes sensor giving it a much higher response and greater sensitivity (see Table 1). On the waxy apple fruit surfaces, unlike on leaf surfaces, the sprayed water was always held in the form of discrete drops, rather than a uniform film over the surface. This in turn meant that, unlike the leaf sensors, fruit wetness sensors did not give a consistent 
full scale response to total wetting. Both types of sensors overestimated the persistence of surface wetness compared with the direct observations of water loss, indicating that the sensors themselves may act as water traps, discussed more fully below. On average, the bare electrode sensors overestimated wetness duration by $5-10 \mathrm{~min}$, while the spiral-bound rubber sensors overestimated by $20-30 \mathrm{~min}$.

Both sensor and water-mass profiles showed irregularities in shape resulting from the formation of water drops on the surface. If enough water was applied, drops coalesced becoming heavy enough to slide down over the sensors and off the fruit. Previous wetting events were found to affect the distribution of water on the apple surface, with more extensive coalescing of droplets, and with these larger droplets tending to adhere better to the apple surface. Fig. 4 shows sensor profiles for a sequence of wetting events. Water droplets collecting on the sensors themselves caused irregular shaped drying profiles. In the first wetting, the apple surface was lightly sprayed, distributing the water evenly over the surface in small droplets. The drying time was short, and the time taken for both types of sensors to return to their original dry value was equal to the drying time determined by direct weighing. When the same samples were subsequently sprayed again, this second wetting was less uniform, with the droplets tending to coalesce and resembling a heavy wetting. This time, although the profiles of the sensor outputs were similar, only the parallel bare-electrodes sensor recorded a similar drying time to the balance output. The spiral-bound rubber sensor overestimated the drying time by $45 \mathrm{~min}$.

There is a greater chance of water accumulation between the spiral-bound rubber sensor and the fruit surface, compared with the very small surface contact of the bare electrode sensor, but, given the level of the signal when the weight measurement indicated a return to the original weight, it appears that there may have been enhanced conductivity due to other factors. It is possible that the prolonged wetness response given by the sensors is a result of the exudation of acid juices from the fruit surface, although there was no visible damage. The spiral-bound rubber sensor was designed to minimise this pressure damage, yet it was this sensor which kept a 'wet' signal for longest. The complexity of fitting the bare electrode fruit wetness sensors was a disadvantage, but when these sensors damaged the apple epidermis, the damage was more likely to be visible than damage from the elastic fitting of the spiral-bound sensors. The greater contact area and the uniformity of pressure on the fruit surface of the bare electrode sensor could lead to less micro-scale damage than the multiple pressure points of the spiral-bound electrodes, and, in practice, the additional care which must be taken to ensure a good fit with the bare wire sensor may also minimise the damage to the fruit surface.

\subsection{Trunk surface wetness}

Unlike living leaves and fruit surfaces, trunk and branch surfaces do not regulate water exchanges with the surrounding atmosphere (James et al., 1984). Bark absorbs or entraps water when ambient humidity is high or free water is deposited on it. The measurement of surface wetness is further complicated by the surface roughness, which reduces the contact area of electrodes and confers a high likelihood of localised water accumulation. The presence of water may be detected erratically by sensors, as the water flows down the trunk or the bark contracts and swells with its changing hydration. The parallel bare electrode sensors tested here gave good surface contact and were not limited by different flow patterns down the trunk because they completely encircled the trunk sample.

Visual assessments were not used to assess the performance of trunk wetness sensors, because of the sub-surface retention of water by the trunk, but the use of the bare electrodes encircling the trunk is an excellent approach, giving good agreement with the water mass measurements.

The onset response of the sensors increased reasonably linearly with the water loading of the sampled trunk ( $r=0.9$ for six single-wetting trials), a relationship that was not observed for fruit and leaf sensors. Sensor responses were close to $0 \mathrm{mV}$ when the sampled trunk was dry, and rose to greater than $1 \mathrm{~V}$ when the trunk sample was visibly wet on the surface. When there was no visible surface water film but the weight measurements indicated that the bark was still hydrated or had water entrapped in its surface texture, intermediate signal responses were obtained.

The agreement between the times for the trunk wetness sensors and the water mass measurements to return to their original dry-sample value was generally 
a.

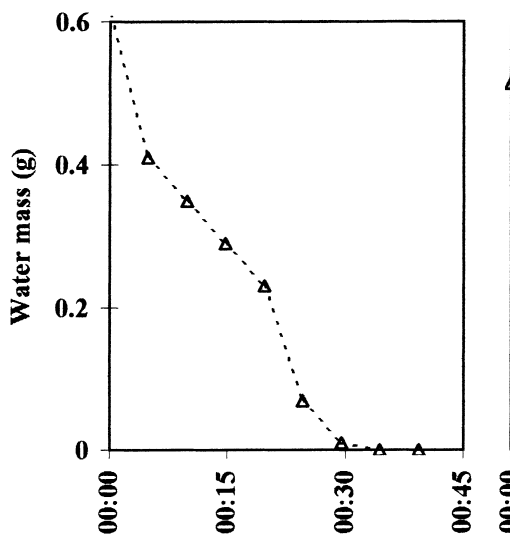

b.
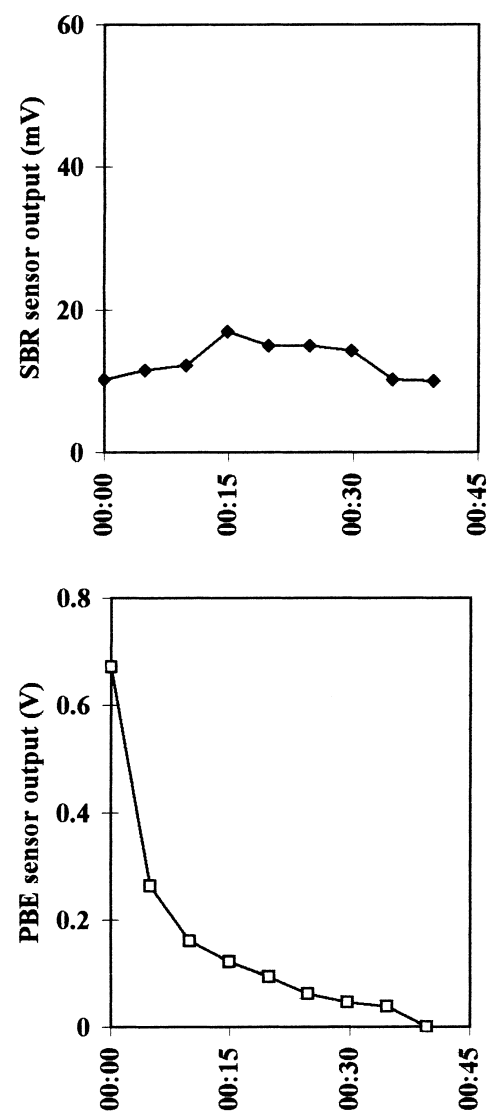
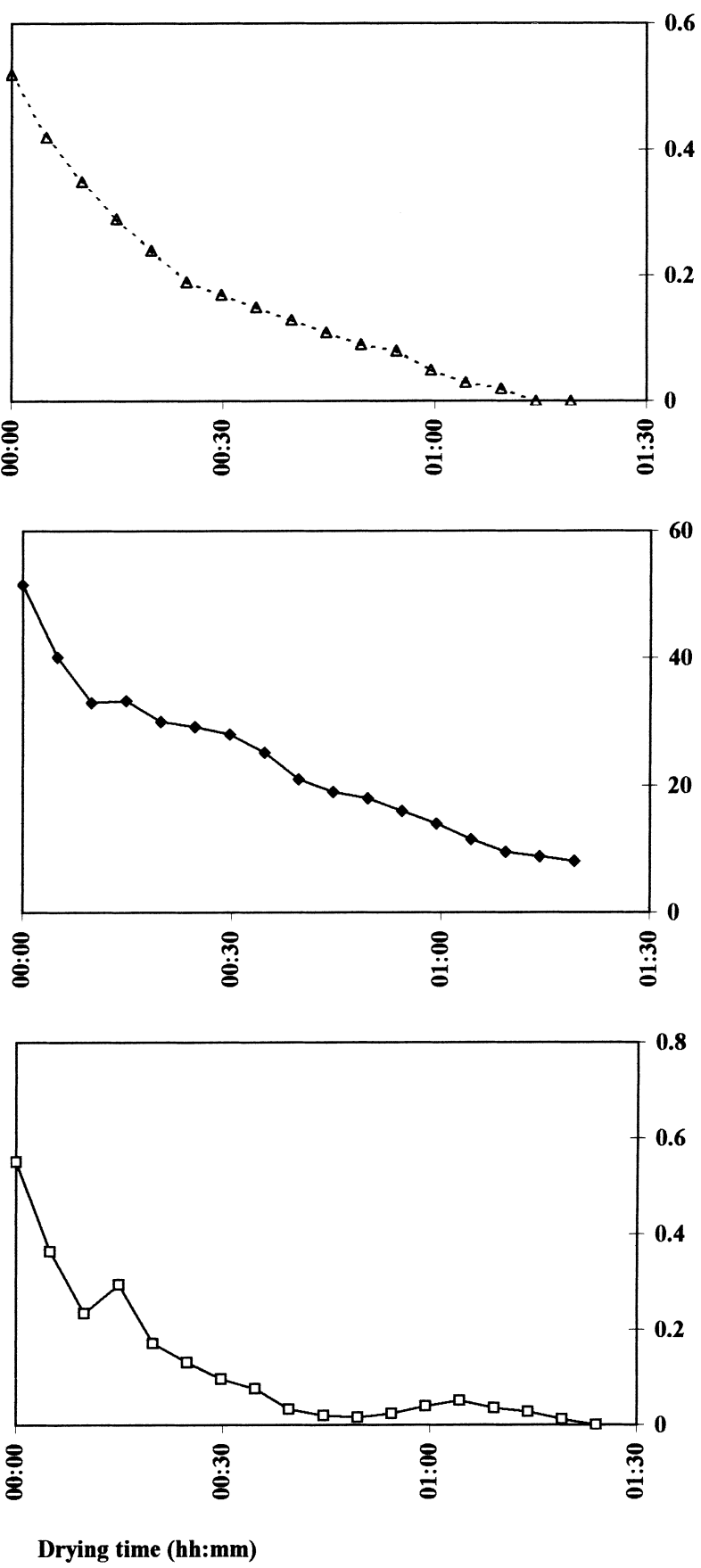

Fig. 4. Typical drying profiles for a fruit wetness trial consisting of two sequential wetting events. The first wetting resulted in small droplets distributed evenly over the apple surface. On rewetting with the same amount of water, droplets coalesced giving a more uneven wetness distribution. (a) Shows measured water mass loss; (b) the spiral bound rubber sensor output; (c) output from a parallel bare electrode sensor. 


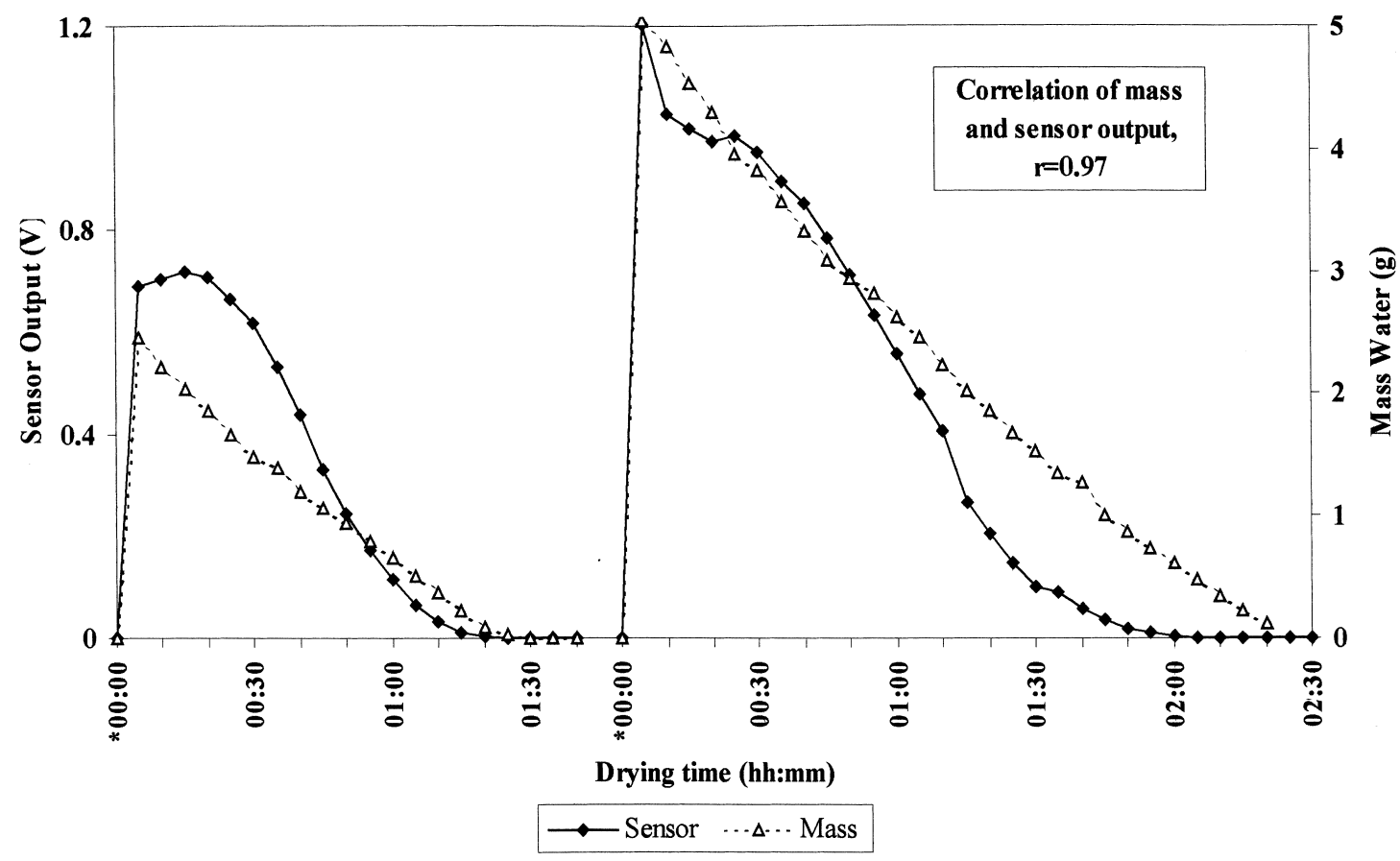

Fig. 5. Sensor response and water mass loss drying profiles for two successive wetting trials of a sample apple trunk. Asterisks show when water was applied.

good. Correlations between the mass of water on the sample and the voltage output were high. The trunk sensor gave S-shaped drying profiles, compared with a near-linear water-mass profile, because of the surface characteristics of the bark and the flow of water down the sampled trunk. Drying rates were affected by the wetting history and the water retention rate by the bark in successive hydration cycles. Fig. 5 shows an example of the signal response to sequential wetting events. On the first wetting, water was applied to the dry log, and it streamed over the log surface. The sensor output rose sharply as the water flowed over the sample surface, and then exhibited a plateau at about $700 \mathrm{mV}$ for over $20 \mathrm{~min}$ before steadily declining. This profile is consistent with the rapid absorption or capture of water into the bark (largely as a result of the rough surface texture), followed by the gradual seepage of the water down the trunk past the sensor, maintaining a wet signal for a considerable length of time. On repeat wetting after the sensor had shown a stable dry signal, another period lasting 20-30 min of comparatively stable sensor output was observed before the sensor indicated that the sample was drying. The drying rate after the second application was slightly slower than for the first wetting event. In the other trials, it was observed that the rate of drying increased after longer dry periods, suggesting that this is not an artefact.

\section{Conclusions}

Sensors tested on leaf, fruit and trunk surfaces have appropriate onset sensitivity, giving distinct voltage peaks when water is applied, up to a maximum ' $100 \%$ wetness' response when water fully bridges the exposed electrode surfaces.

The simplest bare wire electrode sensors give good agreement in drying times with direct observations, and can be used as wetness detectors on different plant surfaces, even for trunk surfaces, where surface texture has a marked impact on wetness distribution. The bare wire sensors are robust, less likely to interfere with the drying process of the sampled surfaces, and 
less susceptible to artefacts arising from water accumulation in their structures.

Systematic problems of accuracy (i.e. mismatches between dryness as determined by the sensors' electrical signal and by direct measurement of water mass or direct observation) outweighed intended design advantages for the leaf grid sensors, which had greater surface coverage and signal sensitivity; and for the spiral-bound rubber fruit wetness sensor.

\section{Acknowledgements}

The authors gratefully acknowledge the technical help provided by ENV's electronics support staff, and thank two anonymous reviewers for their constructive comments on the manuscript.

\section{References}

Aboal, J.R. et al., 1999. The measurement and modelling of the variation of stemflow in a laurel forest in Tenerife, Canary Islands. J. Hydrol. 221 (3/4) 161-175.

Armstrong, R., Barthakur, N.N., Norris, E., 1993. A comparative study of three leaf wetness sensors. Int. J. Biometeorol. 37 (1), $7-10$.

Barthakur, N.N., 1985. A comparative study of radiometric and electronic leaf wetness sensors. Agric. For. Meteorol. 36 (1), 83-90.

Barthakur, N.N., 1987. Comparisons of beta-ray and electronic leaf wetness sensors in a dew chamber. Int. J. Biometeorol. 31 (1), 57-63.

Chakraborty, S., Ratcliff, D., McKay, F.J., 1990. Anthracnose of Stylosanthes scabra: effect of leaf surface wetness on disease severity. Plant Disease 74 (5), 379-384.

Cornell, S.E., 1996. Dissolved organic nitrogen in rainwater, Ph.D. Thesis. University of East Anglia, Norwich, UK, 245 pp.

deWolf, E.D., Francl, L.J., 1997. Neural networks that distinguish infection periods of wheat tan spot in an outdoor environment. Phytopathology 87 (1), 83-87.

Duthie, J.A., 1997. Models of the response of foliar parasites to the combined effects of temperature and duration of wetness. Phytopathology 87 (11), 1088-1095.

Fisher, D.K., Stone, M.L., Elliot, R.L., 1992. Design and testing of a leaf-wetness sensor. In: Proceedings of the American Society of Agricultural Engineers, No. 92-3545, Nashville, TN, USA, $18 \mathrm{pp}$.

Häckel, H., 1974. Eine electrische Methode zur Messung der Blattbenetzungsdauer unmittelbar am Blatt. Agric. Meteorol. 13, 91-103.
Hartman, J.R., Parisi, L., Bautrais, P., 1999. Effect of leaf wetness duration, temperature, and conidial inoculum dose on apple scab infections. Plant Disease 83 (6), 531-534.

Huber, L., Wehrlen, J.M., 1988. La duríe d'humectation en protection des plantes: measure et modélisation. Def. Vegetale 252, 3-11.

James, T.D., Sutton, J.C., Rowell, P.M., 1984. Monitoring wetness of dead onion leaves in relation to Botrytis leaf blight. In: Proceedings of the British Crop Protection Conference, Vol. 2, pp. 627-632.

Leclerc, M.Y., Thurtell, G.W., Gillespie, T.J., 1985. Laboratory simulation of evaporation of water droplets on artificial soybean leaves. Agric. For. Meteorol. 36 (2), 105-111.

Miranda, R.A.C., Butler, D.R., 1986. Interception of rainfall in a hedgerow apple orchard. J. Hydrol. 87, 245-253.

Penrose, L.J., Nicol, H.I., 1996. Aspects of microclimate variation within apple tree canopies and between sites in relation to potential Venturia inaequalis infection. NZ J. Crop Hortic. Sci. 24 (3), 259-266.

Rudgard, S.A., Butler, D.R., 1987. Witches' broom disease on cocoa in Rondônia, Brazil: pod infection in relation to pod susceptibility, wetness, inoculum and phytosanitation. Plant Pathol. 36, 512-522.

Sutton, J.C., Gillespie, T.J., Hildebrand, P.D., 1984. Monitoring weather factors in relation to plant disease. Plant Disease 68, 78-84.

Sutton, J.C., Gillespie, T.J., James, T.D.W., 1988. Electronic monitoring and use of microprocessors in the field. In: Kranz, J., Rotem, J., (Eds.), Experimental Techniques in Plant Disease Epidemiology, pp. 99-113.

Thakur, V.S., Khosla, K., 1999. Relevance of Mills infection periods to apple scab (Venturia inaequalis) prediction and rescheduling fungicide applications in Himachal Pradesh. Ind. J. Agric. Sci. 69 (2), 152-156.

Ward, S.E., 1988. Wetness monitoring in crops, M.Sc. Thesis. Bristol University, Bristol, England, 142 pp.

Wei, Y.Q., Bailey, B.J., Stenning, B.C., 1995. A wetness sensor for detecting condensation on tomato plants in greenhouses. J. Agric. Eng. Res. 61 (3), 197-204.

Weiss, A., Hagen, A.F., 1983. Further experiments on the measurements of leaf wetness. Agric. Meteorol. 29, 207-212.

Weiss, A., Lukens, D.L., 1981. Electronic circuit for detecting leaf wetness and comparison of two sensors. Plant Disease 65, 41-43.

Weiss, A., Lukens, D.L., Steadman, J.R., 1988. A sensor for the direct measurement of leaf wetness: construction techniques and testing under controlled conditions. Agric. For. Meteorol. 43, 241-249.

Wilson, T.B., Bland, W.L., Norman, J.M., 1999. Measurement and simulation of dew accumulation and drying in a potato canopy. Agric. For. Meteorol. 93 (2), 111-119.

Zehr, E.I., Shepard, D.P., Bridges, W.C., 1996. Bacterial spot of peach as influenced by water congestion, leaf wetness duration, and temperature. Plant Disease 80 (3), 339-341. 